\title{
DEVELOPPER DES COMPETENCES CIVIQUES : UN JEU DE PISTE DANS LES CHAMPS DISCIPLINAIRES
}

\author{
(DEVELOPING CIVIC COMPETENCES: \\ A TREASURE HUNT THROUGHOUT THE CURRICULUM)
}

MARIE-FRANCE MAILHOS 1

AEDE-France

\begin{abstract}
In this article, we will show how the development of the civic competences called forth by European Citizenship, can be achieved at school through all the various subject-matters of the curriculum and does not belong exclusively to 'civic education'. Because subject-matters are not identified in the same way across the Member States, we have chosen to regroup them under generic headings: 'scientific', 'linguistic and cultural', 'artistic' and 'conceptual'.

This paper is not an attempt at showing that some sections of the curriculum are better adapted than others to the development of an autonomous critical mind; it is a rambling quest across the curriculum to bring to light the wonderful opportunities for opening the minds of our students and for honing their judgement, whatever subject we may teach.
\end{abstract}

Key words: Citizenship education, critical thinking, debating skills, cultural and intercultural understanding.

\section{Résumé}

Dans cet article, nous allons montrer comment le développement, à l'école, des compétences civiques nécessaires à l'épanouissement d'une citoyenneté européenne n'est pas le domaine exclusif d'une discipline, comme par exemple l'éducation civique, mais peut être réalisé dans n'importe quel champ disciplinaire. Dans les pays européens, les disciplines scolaires sont souvent définies différemment. C'est pourquoi, par souci de lisibilité transeuropéenne, nous avons choisi de regrouper nos réflexions autour de 4 grands domaines disciplinaires : approches scientifique, linguistique et culturelle, artistique et conceptuelle.

Nous n'avons nullement l'intention de montrer que certains domaines disciplinaires sont plus efficaces que d'autres quand il s'agit de développer une pensée autonome et critique ; nous voulons tout simplement effectuer un parcours parmi les savoirs scolaires pour mettre en lumière les magnifiques opportunités pour ouvrir les esprits de nos élèves et affiner leur jugement, quelle que soit la discipline enseignée.

Mots clés : Education du citoyen, pensée autonome et critique, compétences délibératives, compréhension culturelle et interculturelle.

\footnotetext{
1E-mail: mf.mailhos@wanadoo.fr
} 


\section{Les disciplines scientifiques}

Elles permettent de développer un certain mode d'approche du monde. La démarche scientifique repose sur l'observation du réel, accompagnée d'un questionnement pour dépasser les apparences de surface ; la formulation d'hypothèses, l'expérimentation contrôlée, la confrontation des interprétations; et, au final, le respect de conditions rigoureuses pour la validation des conclusions.

L'éducation scientifique des jeunes leur donne des outils pour aborder le réel avec curiosité et lucidité. L'exigence de rigueur qu'elle suppose devrait leur permettre de ne pas se laisser prendre par les discours à la mode, les déclarations hâtives, caricaturales et stéréotypées diffusées par certains médias et par les réseaux sociaux. Entrainés à remettre en question l'évidence et à comparer les solutions proposées en fonction de critères scientifiques, les citoyens devraient être mieux armés pour effectuer des choix, pour les justifier et pour agir en conséquence.

Bien sûr, les disciplines scientifiques apportent aussi des savoirs, indispensables à toute réflexion sur le monde moderne : la biogénétique, les recherches sur les énergies renouvelables, les nanotechnologies et les procédés de plus en plus subtils de traçage et de marquage. Mais beaucoup plus encore que les savoirs sur l'état actuel des connaissances dans ces domaines, il est important que les jeunes aient développé suffisamment leur capacité de questionnement et d'investigation pour aller eux-mêmes à la recherche de nouvelles données, une fois qu'ils auront quitté le système scolaire, et qu'ils sachent les évaluer avec toute la rigueur des protocoles scientifiques.

Pour illustrer ce propos, prenons l'exemple d'activités mises en œuvre dans les modules européens : les débats sur les énergies renouvelables et les choix énergétiques des différents pays représentés ainsi que la question de la mise en place d'une politique européenne sur cette question. Chaque participant a dû, préalablement au débat, faire des recherches sur la situation dans son propre pays et étudier quelques publications présentant des points de vue opposés ; puis, répartis en 6 groupes de 2 ou 3 , représentant des entités variées : consommateurs ordinaires, ingénieurs civils et militaires, fournisseurs d'électricité, groupes écologiques, élus locaux, députés européens, ils ont préparé leur argumentation. Le but n'était pas d'arriver à une conclusion sommaire et catégorique, mais bien de mettre en évidence la complexité du problème et la nécessité d'une démarche reposant sur l'éducation de chacun, la mutualisation des solutions et l'obligation d'une vision européenne inscrite dans un contexte mondial plus ou moins stable.

Dans le domaine de l'énergie comme dans les autres domaines, les élèves d'aujourd'hui, adultes de demain, devront être capables de suivre les évolutions des sciences et des technosciences qui affectent le fonctionnement des sociétés, afin de discerner ce qui apporte des améliorations de ce qui peut devenir une menace pour l'intégrité de l'humain. Pour ce faire, il sera aussi nécessaire qu'ils aient acquis des compétences dans le domaine de la recherche et du traitement de l'information : tout document, toute publication doit pouvoir être soigneusement identifié. 


\section{Les disciplines linguistiques et culturelles}

Dans les disciplines linguistiques, nous englobons non seulement les langues étrangères, mais également la langue maternelle et la langue de scolarisation. En quoi ces enseignements peuventils contribuer à l'éducation du futur citoyen européen ? A cette question, on peut apporter plusieurs types de réponses, tant les champs d'application en sont riches et multiples : accéder au patrimoine commun de la connaissance ; comprendre la langue des voisins européens et découvrir leurs cultures; apprendre à décoder, à décrypter les messages et les textes; apprendre les techniques de la négociation, de l'argumentation et du débat; être capable de reconnaitre l'utilisation sincère du langage et sa perversion...

Accéder au patrimoine commun de la connaissance : savoir lire et entendre une ou plusieurs langues est le moyen d'accéder en toute autonomie au trésor mondial des savoirs. II conviendrait de développer ces capacités dans un minimum de 3 langues appartenant à des 'familles linguistiques' différentes, de façon à bénéficier d'un accès direct à un nombre assez varié de documents originaux, par-delà les subtilités de traduction et, surtout, en dépit de certaines restrictions ou censures plus ou moins avouées ici ou là. A partir de ces 3 langues-racines, un travail sur les familles de langues permet de donner des clés pour la compréhension des langues voisines et désamorce la peur de l'inconnu, en développant la confiance dans la capacité de chacun à surmonter les obstacles linguistiques. Les enseignants de toutes disciplines ne devraient pas hésiter à utiliser dans leurs cours, de temps en temps, des documents en 'version originale', adaptés, bien sûr, à ce que les élèves sont en mesure d'appréhender, pour les familiariser avec cet exercice d'exploration du sens.

Comprendre la langue des autres pays européens et découvrir leurs cultures. Apprentissage de quelques langues : au moins deux en plus de la langue de scolarisation dans la majorité des pays de l'union. Cet apprentissage approfondi donnera l'occasion de faire réfléchir les élèves sur les liens intimes entre langues et cultures et stimulera leur curiosité pour appliquer cette réflexion dans d'autres lieux et d'autres temps.

À ces langues 'naturelles', nous ajouterons évidemment le langage informatique et les techniques de recherche sur Internet, qui nécessitent aussi une éducation dès la formation initiale.

Apprendre à décoder, à décrypter les messages et les textes. L'analyse linguistique, structurée par les théories de la linguistique énonciative interactive et de la pragmatique fournit des outils utiles : qui parle ? À qui ? Dans quel contexte ? Avec quelles intentions ? Comment accéder à l'implicite? Toutes ces questions doivent être posées dès que l'on se trouve face à un 'texte', qu'il soit oral, écrit ou iconographique, publié sur papier ou sur internet, 'posté' sur Facebook, Twitter ou toute autre plateforme. Sans vouloir faire de tous les enseignants des spécialistes de linguistique, il est nécessaire de leur apporter un certain nombre de repères qui leur permettront d'aider leurs élèves à décoder les messages.

II faut mettre en évidence le fait qu'aucun 'texte' ou 'artefact' n'est jamais, comme on le prétend quelque fois, 'objectif'. La notion d'objectivité est un leurre qu'il faut dénoncer le plus rapidement possible. Tout message, quel qu'il soit, est forcément ancré dans une subjectivité. Il a toujours été produit par quelqu'un, dans certaines circonstances, avec une certaine intention. Ce qui est vital, c'est d'être capable d'identifier toutes les références de l'ancrage énonciatif. Une fois ces références mises à jour, alors le texte peut être étudié, analysé et entendu, par rapport à la perspective dans laquelle il fut produit. C'est la seule manière de se prémunir contre la perversion du langage et le mensonge institutionnel, si bien illustrés par Orwell dans 1984; mensonge 
institutionnel d'autant plus perfide et dangereux aujourd'hui qu'il peut être véhiculé, en mots et en images, à travers le monde entier sur les réseaux sociaux. Il est intéressant de signaler ici le travail réalisé par le Conseil de l'Europe pour aider à démasquer les discours de haine et à y apporter remède : No Hate Speech Campaign => http://www.nohatespeechmovement.org/

Développer les compétences de communication et d'interaction ; apprendre les techniques de la négociation, de l'argumentation et de la délibération. II existe de nombreuses méthodes dans ce domaine; nous avons, dans nos stages ELICIT-PLUS, beaucoup utilisé les techniques proposées par E. de Bono. Les cours de langues se prêtent particulièrement bien à cet entraînement, puisque les thèmes abordés et la nature des documents utilisés sont d'une infinie variété et, en général, laissés au libre choix des enseignants : en effet, dans les programmes officiels, seuls les éléments purement linguistiques font l'objet d'instructions ministérielles, les objets culturels choisis pour effectuer ces apprentissages n'étant pas codifiés. Le 'cours' de langue est le lieu idéal où l'on peut aborder des questions sous des perspectives inhabituelles, étrangères ; où l'on peut faire découvrir et apprécier le point de vue du voisin.

\section{Les disciplines artistiques}

Elles affinent la perceptivité et la capacité à interpréter le monde ; elles donnent accès à un langage intime et universel à la fois et c'est précisément cette alchimie qui permet, mieux que toutes autres, le métissage interculturel. Qu'il s'agisse de musique, de cinéma, de danse, de poésie, de peinture ou d'architecture, l'expression artistique donne à voir ou à entendre une représentation transcendée de l'homme dans le monde. Les psychanalystes utilisent d'ailleurs souvent ce type de médium pour accéder à l'indicible de l'humain. La créativité et l'imagination suggèrent souvent des solutions inattendues aux problèmes.

Un minimum de connaissances d'histoire des arts, des différentes 'écoles' qui ont façonné l'expression artistique en Europe est nécessaire aux enseignants de toutes disciplines pour mieux comprendre leur environnement et aider les élèves à découvrir leur héritage et à y contribuer ; voir Loyer (2017) et les multiples facettes de la culture européenne. Pour les enseignants de disciplines artistiques, l'étude contextualisée du patrimoine européen et des créations actuelles doit fournir l'occasion de mettre en évidence des points de vue singuliers à partir desquels il est possible de comprendre une histoire commune et des valeurs partagées.

L'architecture n'étant pas une discipline scolaire, où trouvera-t-elle sa place dans le programme des élèves ? Dans le cours de langues, bien sûr, mais aussi en géographie, 'aménagement de l'espace urbain', en histoire ou en sciences économiques et sociales. Une première question aurait pour objectif de faire apparaître la valeur symbolique de l'architecture institutionnelle. On peut ensuite faire apparaitre, à travers l'étude de cartes postales de paysages urbains, la similitude des préoccupations et des enjeux, par-delà les différentes manières d'envisager la vie quotidienne aux quatre coins de l'Europe.

Une photo de terrain vague peut être à l'origine d'un débat : simuler une réunion de conseil municipal visant une décision concernant l'utilisation future d'un terrain vague, en plein centre-ville, suite à la démolition d'un vieux quartier. II ne s'agit pas ici à proprement parler d'une décision à dimension européenne, mais bien de pratiquer le 'consensus procédural minimum' dans un groupe multinational et multiculturel dont parle Davidson (1996), chaque participant apportant à la discussion son expérience locale nourrie de son histoire et de ses convictions. Plus importante encore que la discussion elle-même est le retour réflexif sur le fonctionnement du groupe, les 
modalités de prise de parole, les obstacles et les éléments facilitateurs : un exercice de prise de conscience du développement de compétences délibératives et de stratégies de prise de décision, centrales à l'éducation du futur citoyen européen qui va découvrir ainsi la diversité des pratiques sociales. C'est ainsi que les expériences vécues dans le cadre du 'Parlement européen des Jeunes' et du dispositif 'EUROSCOLA' sont particulièrement fructueuses.

\section{Les disciplines conceptuelles : histoire, éducation civique, politique, sociale, religieuse et philosophique}

Nous entendons ici les disciplines qui abordent les systèmes conceptuels théoriques, c'est-àdire ceux qui sont construits entièrement par l'esprit humain, prenant appui sur des objets concrets. Ces systèmes intellectuels ont un impact fort sur les fonctionnements collectifs et sur les attitudes individuelles ; il s'agit du système des institutions, des organisations sociétales, des croyances religieuses, des courants philosophiques. Dans les parcours scolaires des pays de l'union, ces domaines sont abordés dans des disciplines variées que chacun pourra identifier.

C'est principalement dans le champ de ces disciplines que les enseignants vont pouvoir faire découvrir à leurs élèves l'évolution de l'idée de démocratie, que ce soit dans les cours d'histoire, d'études politiques et juridiques, en éducation civique, en cours d'éducation au fait religieux, ou en philosophie.

Les grands courants de pensée qui ont traversé l'Europe depuis l'antiquité et qui ont façonné notre paysage intellectuel ne s'arrêtent jamais aux frontières d'un état et l'histoire, au sens le plus large du terme, doit rendre compte de ces vastes mouvements et des cocktails variés qu'ils ont produits en rencontrant les spécificités locales.

Pourquoi placer l'histoire ici ? En raison des biais idéologiques qui déforment la vérité des faits. Au pire, on trouve le négationnisme et le révisionnisme. Au mieux, dans les programmes scolaires, I'histoire est présentée sous un angle national. On peut s'amuser à comparer comment est traitée la période napoléonienne dans les manuels scolaires des États Membres : de la Pologne à Malte, en passant par la Suède et l'Espagne, les récits sont en effet, contrastés. II serait donc tout à fait éclairant, pour l'éducation du futur citoyen européen, de susciter sa curiosité et de l'aider à découvrir comment les différentes perspectives nationales colorent différemment les faits historiques. Les histoires nationales ne peuvent pas se comprendre si l'on reste à l'intérieur des frontières ; il faut prendre de la distance et se regarder d'ailleurs, se regarder autrement. A ce propos, il faut citer la publication du conseil de l'Europe : Crossroads of European Histories (2006) qui présente des points de vue variés sur cinq moments clés de l'histoire de l'Europe: 1848, 191213, 1919, 1945 et 1989. Dans chacun de ces chapitres, un panel d'historiens de différents pays européens expose les points de vue nationaux sur le même évènement. Cette volonté de 'multiperspective', adoptée par le conseil de l'Europe dès 1990, constitue une avancée particulièrement significative dans la construction des " points de convergence » indispensables au « vivre ensemble » des citoyens européens. De même, les romans, ou les films, qui présentent des points de vue spécifiques, en contrepoint ou en miroir, peuvent apporter des éclairages originaux qui enrichissent notre compréhension. Dans un récent stage ELICIT-PLUS (Strasbourg, novembre 2016), nous avons fait travailler les groupes d'enseignants européens sur notre atelier "People on the Move; A History of Europe ». L'une des activités visait à retrouver comment est traitée la question des migrations dans les manuels scolaires des différents pays représentés: étude quantitative et qualitative dont le résultat se passe de commentaires. 


\section{Conclusion}

Pour construire une conscience civique du citoyen européen, il faut trouver les «biens communs " qui permettent d'élaborer le cadre politique dans lequel cette citoyenneté pourra s'exprimer et imprimer sa volonté de «revitaliser la démocratie» (Davidson, 1996). Pour trouver les indispensables «biens communs», il faut apprendre aux futurs citoyens à « se rendre attentifs» aux «points d'entrecroisement», qui «ne restent eux-mêmes des carrefours vifs que si chacun apporte ses convictions, mais aussi les sources à partir desquelles il les alimente.» (Ricœur, 1995).

Apprendre aux jeunes enseignants à identifier les « sources de leurs convictions ", les valeurs sous-jacentes de tous les actes éducatifs et pédagogiques de leurs pratiques professionnelles quotidiennes; c'est en général un axe fort des curriculum, dans les instituts de formation d'enseignants. Pour que cette réflexion atteigne une dimension européenne, il est important de permettre aux enseignants de se rencontrer et de comparer leurs pratiques et les principes qui les sous-tendent, en travaillant ensemble sur des objets professionnels.

\section{Références}

Angel, B. \& Lafitte J. (1999). L'Europe, petite histoire d'une grande idée. Paris: Gallimard, coll. Découvertes.

Berthoud, A.C. (1996). Paroles à propos, approche énonciative et interactive du topic. Paris: Ophrys.

Davidson, A. (1996). Rethinking Human Rights, The Citizen who does not Belong: Multiculturalism, Citizenship and Democracy. Annandale: The Federation Press.

De Bono, E. (1985). Six Thinking Hats. London: Penguin Books

Dudley J., Robinson, J. \& Taylor, A. (1999). Educating for an Inclusive Democracy: Critical Citizenship Literacy. Discourse: Studies in the cultural Politics of Education, 20(3), Carfax Publishing. pp. 427-441. Retrieved from https://doi.org/10.1080/0159630990200306

Loyer, E. (2017). Une brève histoire culturelle de l'Europe. Paris: Flammarion.

Stanley, D. (2006). A Reflection on the Function of Culture in Building Citizenship Capacity, Strasbourg: Council of Europe Publishing.

Stradling, R. (ed.) (2006). Crossroads of European Histories. Strasbourg: Council of Europe Publishing. 